\title{
Visual imagery versus visual experience of familiar individuals
}

\author{
DAVID J. BRYANT \\ Stanford University, Stanford, California
}

(Gordon H. Bower, Sponsor)

\begin{abstract}
Subjects rated the physical similarity of familiar individuals from photographs and mental images. Multidimensional scaling and hierarchical clustering techniques revealed little difference between photographs and mental images in the subjects' similarity ratings. This result implies that people's preexisting imaginal representations derived from direct experience are functionally equivalent to their visual percepts of human beings.
\end{abstract}

The relation between mental imagery and visual experience has been an interesting puzzle to psychologists for quite some time. Research has demonstrated that visual properties such as size (Kosslyn, 1976), shape (Shepard \& Chipman, 1970), and color (Finke \& Schmidt, 1977) can be represented in people's mental images. Furthermore, studies have demonstrated the functional equivalence of mental images and visual percepts in such tasks as dot localization (Podgorny \& Shepard, 1978) and rotation of forms (Shepard \& Metzler, 1971). Such demonstrations imply that imagining an object is similar to the experience of seeing it (for reviews regarding the equivalence of imagery and perception, see Finke, 1980, and Finke \& Shepard, 1986). Some evidence, however, suggests that mental imagery is related to higher order cognitive processes, and that it differs from visual perception. For example, Kosslyn and associates have found that people construct mental images by parts (Kosslyn, Ball, \& Reiser, 1978; Kosslyn, Reiser, Farah, \& Fliegel, 1983), and congenitally blind individuals perform on imagery tasks in a fashion similar to how sighted individuals perform (Kerr, 1983). Thus, the relation between visual and imaginal experience continues to be a puzzle.

An important issue in this debate is to what extent the representations people use to form mental images of objects are like the representations they use in perceiving those objects (Farah, 1988). A feature of many studies in this area is that subjects have judged stimuli that were relatively unfamiliar to them (e.g., Kosslyn, 1976; Podgorny \& Shepard, 1978; Shepard \& Metzler, 1971). Given that subjects are asked to imagine things they have only recently experienced, researchers may have been

I am grateful to Roger Shepard for his invaluable assistance in the completion of this work. I would like to thank the 15 people who allowed their photographs to be used as stimuli, and Caren Jones for her helpful comments. Correspondence should be addressed to David Bryant, Department of Psychology, Stanford University, Stanford, CA 94305.

Electronic mail may be sent to bryant@psych.stanford.edu. stacking the deck in favor of finding equivalence of images and percepts. To avoid this problem, and to provide a fairer test of the equivalence of imagery and perception, researchers have investigated subjects' imagery for familiar objects, of which subjects presumably have longterm representations. A high degree of correspondence between subjects' similarity ratings based on names and those based on pictures has been found when stimuli were outline figures of states of the United States (Shepard \& Chipman, 1970), faces of political figures (Gordon \& Hayward, 1973), and faces of motion-picture actors (Shepard \& Castro, cited in Shepard, 1975). These results indicate that imagining an object on the basis of a memory representation is functionally equivalent to perceiving that object.

Although Gordon and Hayward (1973) and Shepard and Castro (cited in Shepard, 1975) have examined imagery of familiar people, their stimuli were faces of famous individuals whom the subjects were unlikely to have ever met. Subjects must have learned these faces from photographs, television, and movies, and so the comparison in these studies was made between perceiving pictures of faces and imagining those faces on the basis of one's memory of previous pictures. The purpose of the following experiment was to examine the extent to which the representations that underlie visual imagery are like those underlying visual perception, using multidimensional scaling (MDS) and hierarchical clustering techniques. In this study, however, familiar individuals that the subjects knew personally were selected as stimuli, because they could easily be imagined on the basis of just a name, and the subjects developed their representations of these people from direct experience, rather than pictures.

MDS and clustering techniques provide means for interpreting subjects' similarity ratings of objects. To the extent that subjects' imaginal representations are like their perceptual representations, they should generate equivalent ratings of similarity, whether the subjects are making their judgments on the basis of mental images or visual 
experience. A second-order isomorphism (Shepard \& Chipman, 1970) of imagery and visual perception, reflected in similar MDS and clustering representations of their similarity ratings, would constitute evidence that people's long-term representations of visual information are functionally equivalent to their perceptual representations.

\section{METHOD}

\section{Subjects}

Four graduate students in the Stanford Psychology department served as volunteers in this experiment.

\footnotetext{
Materials

Eight female and 7 male graduate students in the Stanford psychology department served as "experimental individuals." The appearances of the experimental individuals were familiar to the subjects, as assessed by a preexperimental questionnaire. A $5 \times 7$ color photograph was taken of the chest and face of each individual who posed in front of a blank background. An IBM PC was employed to collect the subjects' similarity ratings.

Design

The subjects completed two tasks: the picture rating task, which consisted of rating the similarity of appearances of experimental individuals on the basis of their photographs, and the imagery rating task, which consisted of rating the similarity of appearances of experimental individuals on the basis of mental imagery. During each task, the subjects were presented with either all possible (105) pairs of photographs or names of experimental individuals in random order, so that subjects made a comparison of each possible pair of experimental individuals. The subjects gave ratings of physical similarity on a 10-point scale in which " 9 " indicated maximal similarity and " 0 " indicated maximal dissimilarity. Two subjects completed the imagery rating task first and the picture rating task second, and 2 completed the tasks in the reverse order.
}

\section{Procedure}

During the picture rating task, the subjects sat at an IBM PC while the experimenter presented pairs of photographs according to a random sequence controlled by the computer. The subject indicated a similarity rating as each pair was presented, by typing a number from 0 to 9 on the computer keyboard. During the imagery rating task, the computer presented a random sequence of pairs of names of experimental individuals on the computer screen. The subjects again indicated a similarity rating as each pair was presented.

\section{RESULTS}

The data were analyzed using nonmetric multidimensional scaling (Kruskal, 1964; Shepard, 1962) performed by the KYST computer program (Kruskal, Young, \& Seery, 1973), and by an additive tree-fitting procedure performed by the ADDTREE computer program (Carroll, 1976; Sattath \& Tversky, 1977). Multidimensional scaling represents similarity between objects as spatial proximity of those objects in an $n$-dimensional space, and tree-fitting techniques represent similarity as shortest paths through a tree structure.

The order in which the subjects completed the rating tasks had little impact on either the MDS or the tree-fitting solutions. For this reason, the data were collapsed across task order.

\section{Multidimensional Scaling}

The mean correlation between the subjects' ratings on the imagery and on the picture tasks was .85 . Thus, the subjects' similarity ratings tended to be much the same,

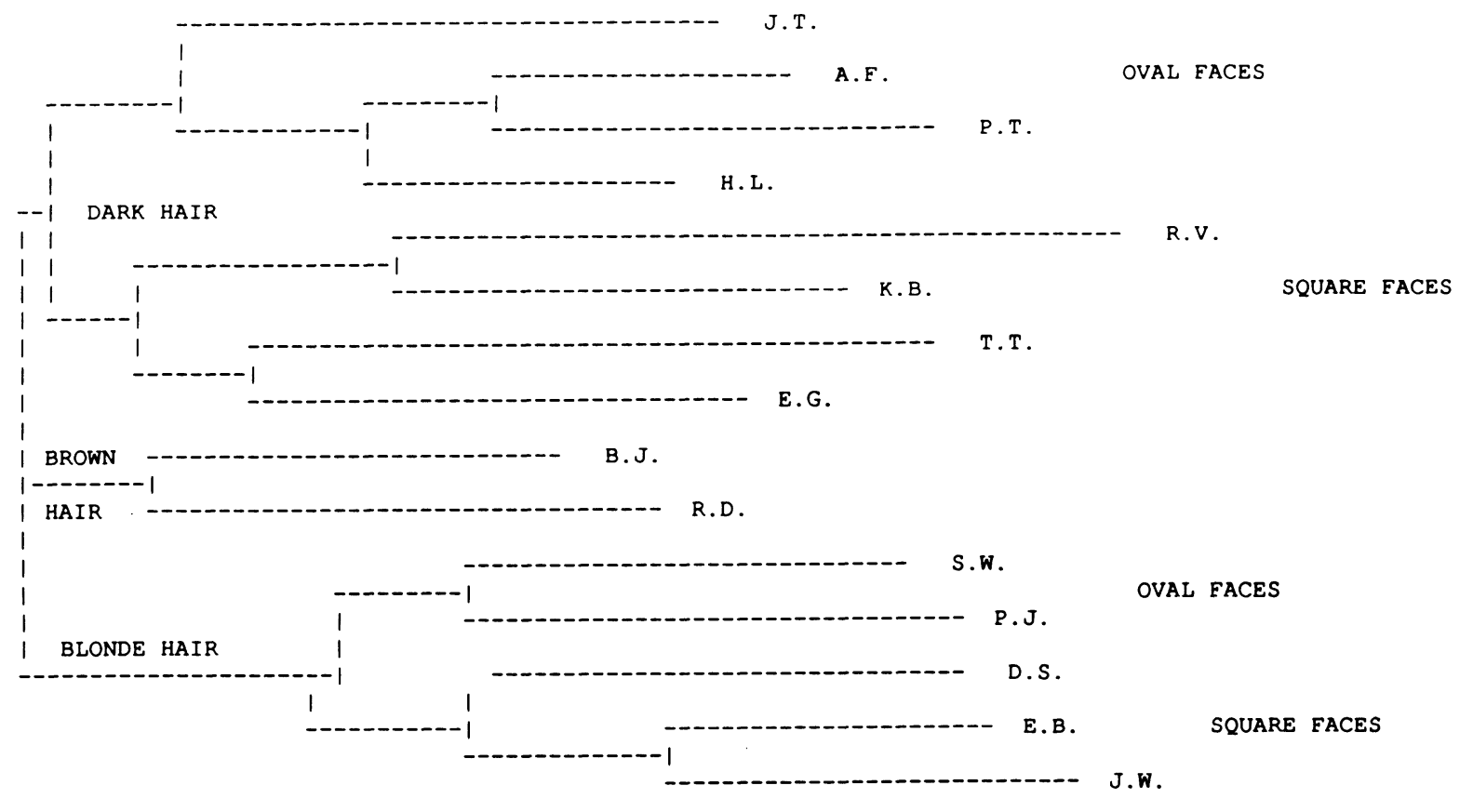

Figure 1. ADDTREE solution for the picture condition. The experimental individuals are identified by contrived initials, and the broad groupings are indicated in block letters. 


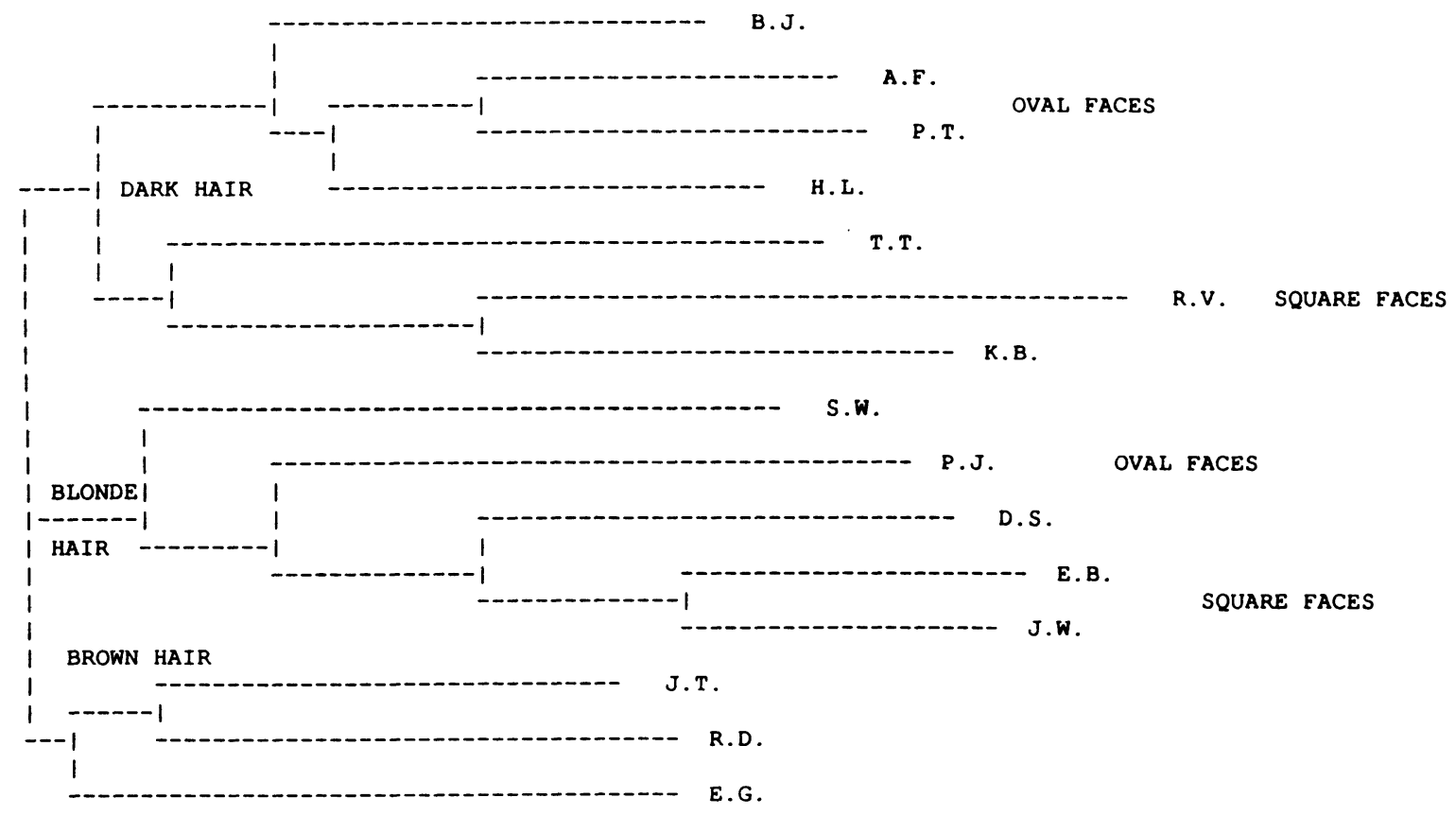

Figure 2. ADDTREE solution for the imagery condition. Experimental individuals are again identified by contrived initials and the broad groupings are indicated in block letters.

whether they were judging pictures or mental images. An MDS procedure, however, was used to further explore the relation of subjects' performance on the two tasks. ${ }^{1}$ For both tasks, three initial configurations of experimental individuals were employed in the KYST program. A solution was considered to be globally optimal if it could be consistently obtained with several starting configurations and had a minimal stress value associated with it. ${ }^{2}$ While solutions in four or five dimensions offered the greatest reduction of stress, these were not readily interpretable, and two-dimensional solutions were accepted for both the imagery and picture rating tasks.

The two-dimensional MDS solutions for the imagery and picture conditions were very similar, and the two dimensions seemed to be similarly interpretable in each case. One was a "color" dimension, ranging from individuals with blonde hair and fair complexions at one extreme, to individuals with black hair and dark complexions at the other. The other dimension seemed to reflect gender-related features, such as hair length, skin smoothness, and face shape, because females tended to be at one pole and males at the other. The subjects were not simply making polar judgments of gender, however, for there was a continuum of individuals along this dimension. In addition, the clustering analysis revealed that face shape seemed to influence the subjects' judgments. Overall, the patterns of the solutions were very similar in the imagery and picture conditions. Thus, the subjects appear to have used the same criteria or dimensions when rating appearance in the imagery and picture conditions, and they seem to have reached similar conclusions.

\section{Tree-fitting}

An additive tree-fitting procedure, ADDTREE, was employed in order to further explore the similarity data and reveal relations between the experimental individuals. The similarity of the ADDTREE solutions for the imagery and picture tasks (see Figures 1 and 2 ) is striking. ${ }^{3}$ With the exception of the transposition of J.T. and B.J. and the movement of E.G. to another branch, all the experimental individuals fall into the same basic clusters. Analysis of the clustering solutions revealed that the subjects appeared to distinguish between oval and square faces. As is labeled in Figure 1, this was true for both dark- and light-haired individuals. The clusters may also be distinguishable on the basis of other features as well, but face shape was the most apparent, and there is evidence that face shape is a salient feature in perception from pictures (Ellis, 1984; Ellis, Deregowski, \& Shepherd, 1975) and imagery (Ellis, Shepherd, \& Davies, 1980).

\section{DISCUSSION}

The functional equivalence of mental imagery and perception has been established in a number of tasks (Finke, 1985). In many of these tasks, however, the stimuli have been relatively novel or learned from pictures, so the subjects may have been biased to display similar perfor- 
mance in imagery and perceptual tasks. There are both ecological and theoretical reasons why one would want to compare imagery and perception of highly familiar objects. When people use imagery in real life, they tend to imagine familiar things for which they have a long-term representation derived from direct experience. In addition, much debate in this area centers on the relation of long-established imaginal representations to perceptual ones (Farah, 1988). Thus, in this experiment, people's imagery of familiar individuals was compared with their perception of photographs of those individuals.

Three major findings arose. First, interpretable MDS and additive tree clustering solutions were obtained for similarity judgments of human appearance, both when subjects employed pictures and when they employed mental imagery. Second, subjects used the same general features to make their ratings when using pictures and when using imagery. In both cases, hair color, gender-related features such as hair length and complexion, and face shape influenced subjects' ratings. Third, subjects exhibited remarkably similar psychological spaces for photographs and mental images of familiar individuals. Their ratings of physical similarity were highly consistent whether they rated photographs or mental images. Thus, imagery and perception of human faces show a secondorder isomorphism, even when subjects form their images from longterm representations. These findings offer evidence that long-term imaginal representations are functionally equivalent to visual percepts.

\section{REFERENCES}

CARRoll, J. D. (1976). Spatial, non-spatial and hybrid models for scaling. Psychometrika, 41, 439-463.

Ellis, H. D. (1984). Practical aspects of face memory. In G. L. Wells \& E. F. Loftus (Eds.), Eyewitness testimony: Psychological perspectives (pp. 12-37). Cambridge, U.K.: Cambridge University Press.

Ellis, H. D., Deregowski, J. B., \& ShePherd, J. W. (1975). Descriptions of white and black faces by white and black subjects. International Journal of Psychology, 10, 119-123.

Ellis, H. D., Shepherd, J. W., \& Davies, G. W. (1980). The deterioration of verbal descriptions of faces over different delay intervals. Journal of Police Science \& Administration, 8, 101-106.

FARAH, M. J. (1988). Is visual imagery really visual? Overlooked evidence from neuropsychology. Psychological Review, 95, 307-317.

FinkE, R. A. (1980). Levels of equivalence in imagery and perception. Psychological Review, 87, 113-132.

FINKE, R. A. (1985). Theories relating mental imagery to perception. Psychological Bulletin, 98, 236-259.

Finke, R. A., \& SCHmidT, M. J. (1977). Orientation-specific color aftereffects following imagination. Journal of Experimental Psychology: Human Perception \& Performance, 3, 599-606.

FinKe, R. A., \& SHEPARD, R. N. (1986). Visual functions of mental imagery. In K. R. Boff, L. Kaufman, \& J. P. Thomas (Eds.), Handbook of perception and performance: Vol. 2. Cognitive processes and performance. New York: Wiley.

GORDON, I. E., \& HAYWARD, S. (1973). Second-order isomorphism of internal representations of familiar faces. Perception \& Psychophysics, 14, 334-336.
KerR, N. H. (1983). The role of vision in "visual imagery" experiments: Evidence from the congenitally blind. Journal of Experimental Psychology: General, 112, 265-277.

Kosslyn, S. M. (1976). Can imagery be distinguished from other forms of internal representation? Evidence from studies of information retrieval times. Memory \& Cognition, 4, 291-297.

Kosslyn, S. M., Ball, T. M., \& Reiser, B. J. (1978). Visual images preserve metric spatial information: Evidence from studies of image scanning. Journal of Experimental Psychology: Human Perception \& Performance, 4, 47-60.

Kosslyn, S. M., Reiser, B. J., Farah, M. J., \& Fliegel, S. L. (1983). Generating visual images: Units and relations. Journal of Experimental Psychology: General, 112, 278-303.

KRUSKAL, J. B. (1964). Multidimensional scaling by optimizing goodness of fit to a nonmetric hypothesis. Psychometrika, 29, 1-27.

Kruskal, J. B., Young, F. W., \& Seery, J. B. (1973). How to use KYST, a very flexible program to do multidimensional scaling and unfolding. Unpublished manuscript, Bell Laboratories.

Podgorny, P., \& Shepard, R. N. (1978). Functional representations common to visual perception and imagination. Journal of Experimental Psychology: Human Perception \& Performance, 9, 21-35.

Sattath, S., \& Tversky, A. (1977). Additive similarity trees. Psychometrika, 42, 319-345.

SHEPARD, R. N. (1962). The analysis of proximities: Multidimensional scaling with an unknown distance function. Psychometrika, 27, $125-140$

SHEPARD, R. N. (1975). Form, formation, and transformation of internal representations. In R. Solso (Ed.), Information processing and cognition: The Loyola symposium (pp. 87-122). Hillsdale, NJ: Erlbaum.

Shepard, R. N., \& Chipman, S. (1970). Second-order isomorphism of internal representations: Shapes of states. Cognitive Psychology, 1, 1-17.

Shepard, R. N., \& Metzler, J. (1971). Mental rotation of threedimensional objects. Science, 171, 701-703.

\section{NOTES}

1. I have not included the MDS solutions because of difficulties in presenting the color photographs, which are important in interpreting the solutions. The solutions and copies of the experimental materials can be made available on request.

2. The stress value is a numeric measure of the badness-of-fit of an MDS solution. Although good fits, and hence lower stress, are desirable, good fit in the absence of interpretability defeats the purpose of MDS - the revelation of hidden structure in data.

3. Distance along vertical line segments does not reflect similarity in an ADDTREE solution. Thus, the inverting of the order of the blonde and brown-haired groups in Figures 1 and 2 does not reflect a difference in the solutions. 